\title{
PROBABILISTIC STUDY OF BONE REMODELING USING FINITE ELEMENT ANALYSIS
}

\author{
C. WERNER and R.S.R. GORLA* \\ Department of Mechanical Engineering \\ Cleveland State University \\ Cleveland, Ohio 44115 USA \\ E-mail: r.gorla@csuohio.edu
}

\begin{abstract}
The dynamic bone remodeling process is a computationally challenging research area that struggles to understand the actual mechanisms. It has been observed that a mechanical stimulus in the bone greatly affects the remodeling process. A 3D finite element model of a femur is created and a probabilistic analysis is performed on the model. The probabilistic analysis measures the sensitivities of various parameters related to the material properties, geometric properties, and the three load cases defined as Single Leg Stance, Abduction, and Adduction. The sensitivity of each parameter is based on the calculated maximum mechanical stimulus and analyzed at various values of probabilities ranging from 0.001 to 0.999 . The analysis showed that the parameters associated with the Single Leg Stance load case had the highest sensitivity with a probability of 0.99 and the angle of the force applied to the joint of the proximal femur had the overall highest sensitivity.
\end{abstract}

Key words: probabilistic analysis, bone remodeling, finite element analysis.

\section{Introduction}

It has been observed that bone has the capacity to repair itself when subject to damage due to loading. The damaged bone is removed by cells called osteoclasts in a process known as bone resorption. The new bone is built in the void by cells called osteoblasts in a process known as bone formation and this entire process is appropriately known as bone remodeling. This natural process is necessary to maintain the skeletal strength required for the human body to function. Several mathematical models have been created to characterize this cyclic phenomenon. If the remodeling process becomes out of balance and more resorption occurs than formation, then the remodeling can lead to a higher porosity and less dense bone which eventually leads to osteoporosis. The imbalance in bone remodeling is also observed in long duration exposure to microgravity environments (i.e., astronauts on board the international space station) and the loss of bone density occurs at a much higher rate than on earth where it is typically observed at later stages of an individual's life.

A popular method to determine the amount of strain a given portion bone undergoes when it is loaded is using the finite element method. This method can reduce complicated geometry of a bone to much smaller geometric shapes that can be mathematically described by their shape functions. The finite element method can identify regions of a bone that experience a higher mechanical stimulus and may be more susceptible to the bone remodeling process. Because bone resorption creates voids in the bone, the local mechanical properties will change where the resorption occurs and redirect the load to a stiffer area which would increase the stimulus in another region. The mechanical properties will be updated in the finite element model through the relationship between the porosity and the modulus of elasticity. This interaction and constant update of the finite element model can be computationally expensive because material properties will have to be assigned at an elemental basis, or at least, for a given region.

Several articles have been written of combining the bone remodeling process with the finite element

\footnotetext{
* To whom correspondence should be addressed
} 
method. Hazelwood created a mechanistic model of bone remodeling using the mechanical stimulus (Hazelwood and Castillo, 2007). The mechanical stimulus is a formula that combines the various strains in the bone and the associated times per day that strain is achieved and has units of cycles per day. Hazelwood's mechanistic model compares the mechanical stimulus to a single value activation frequency to determine if a region of bone will undergo the bone remodeling process. He then applies the mathematical concept of bone remodeling to a 2D finite element model of a femur (Hazelwood et al., 2001). The loads on the finite element model are three separate load cases to define the load history for normal activity. The article than uses the time dependent remodeling process and applies an additional fourth load case to the model which intends to simulate the additional strain from marathon training.

The present paper will create a 3D finite element model based on the CAD geometry of an actual femur and apply the three load cases that define normal activity from Hazelwood's marathon training article. In addition, the parameters that define the loads, properties of the bone, and geometry will be varied to perform a probabilistic analysis using NESTEM and determine the sensitivity of each parameter with a given probability.

\section{Analysis}

The probabilistic analysis is performed by varying 22 different variables in the finite element model and the post processing calculations. Each variable is defined and adjusted + and $-10 \%$ as shown in Tab.1 below

Table 1 . Summary of parameters.

\begin{tabular}{|l|r|rr|}
\hline & \multicolumn{3}{|c|}{ Parameters } \\
\hline Varying Properties & $-10 \%$ & Standard & $+10 \%$ \\
\hline Cortical Modulus (psi) & 2871747.0 & 3190830.0 & 3509913.0 \\
\hline Cortical poisson's ratio & 0.27 & 0.30 & 0.33 \\
\hline Trabecular Modulus (psi) & 130534.2 & 145038.0 & 159541.8 \\
\hline Trabecular poisson's ratio & 0.27 & 0.30 & 0.33 \\
\hline Average Element Size (in) & 0.225 & 0.250 & 0.275 \\
\hline Cortical Thickness (in) & 0.177 & 0.197 & 0.217 \\
\hline Femur Length (in) & 9.3 & 10.3 & 11.3 \\
\hline Single Leg Stance cpd & 4050 & 4500 & 4950 \\
\hline Single Leg Stance Load Joint (lb) & 468.8 & 520.9 & 573.0 \\
\hline Single Leg Stance Angle Joint (deg) & 21.6 & 24.0 & 26.4 \\
\hline Single Leg Stance Load Abductor (lb) & 340.9 & 378.8 & 416.7 \\
\hline Single Leg Stance Angle Abductor (deg) & 25.2 & 28.0 & 30.8 \\
\hline Abduction cpd & 675 & 750 & 825 \\
\hline Abduction load Joint (lb) & 234.3 & 260.3 & 286.3 \\
\hline Abduction Angle Joint (deg) & -13.5 & -15.0 & -16.5 \\
\hline Abduction Load Abductor (lb) & 170.4 & 189.3 & 208.2 \\
\hline Abduction Angle Abductor (deg) & -7.2 & -8.0 & -8.8 \\
\hline Adduction cpd & 675 & 750 & 825 \\
\hline Adduction Load Joint (lb) & 313.2 & 348.0 & 382.8 \\
\hline Adduction Angle Joint (deg) & 50.4 & 56.0 & 61.6 \\
\hline Adduction Load Abductor (lb) & 227.8 & 253.1 & 278.4 \\
\hline Adduction Angle Abductor (deg) & 58.5 & 65.0 & 71.5 \\
\hline
\end{tabular}




\subsection{CAD setup}

The desired bone to be used in this analysis is the proximal femur. Many studies conclude the importance of this bone and areas such as the femoral neck that have been responsible for fractures due to the weakened structure. The femur, provided by SAEC-Kinetic Vision, was created by segmenting data from an actual MRI scan of an individual's femur. The data was refined and surfaced to create a single volume CAD model shown in Fig.1 below

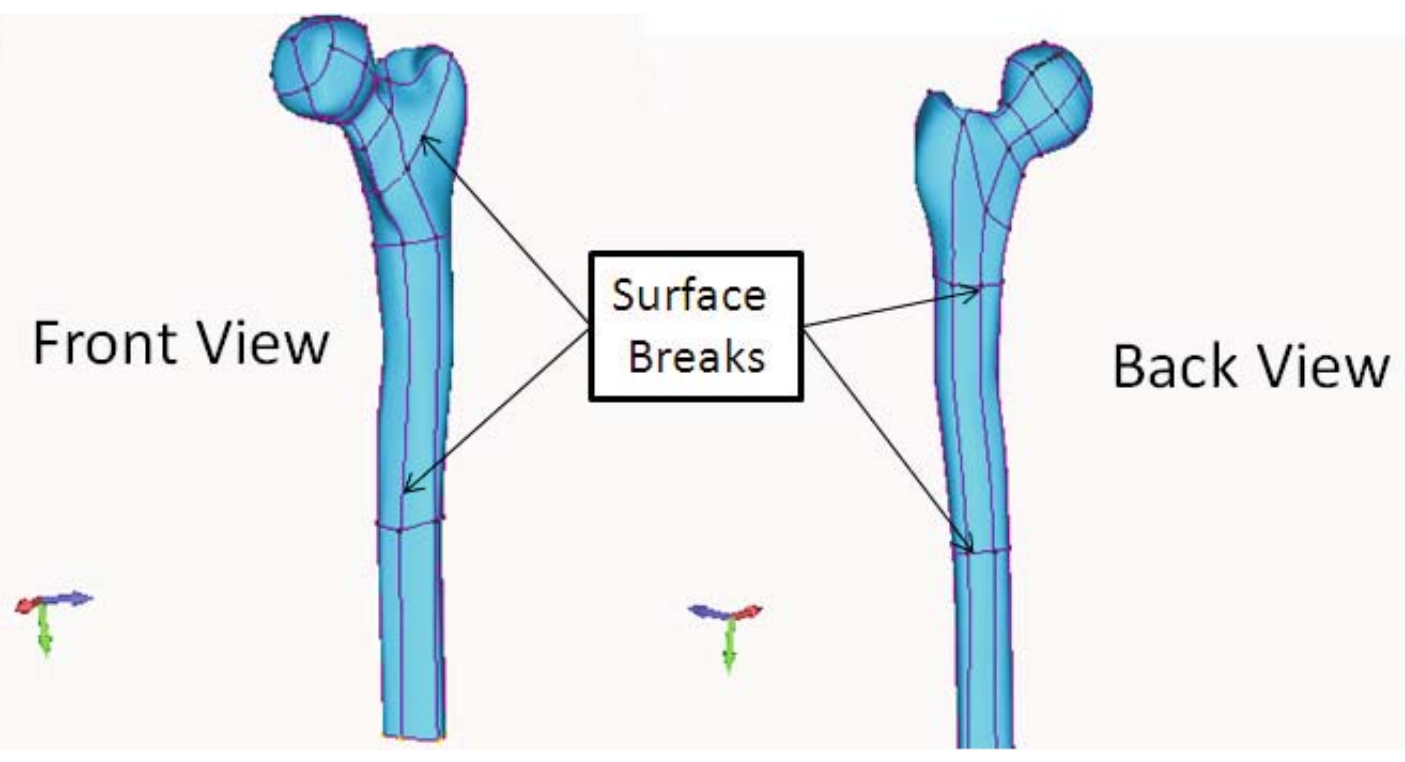

Fig.1. CAD model of proximal femur. Courtesy of SAEC-Kinetic Vision.

This CAD geometry remained constant for the probabilistic analysis with exception of the geometric length parameter. The length of the provided CAD femur does not represent the actual total length of a femur but, rather, an arbitrary portion of it. The length of the femur in the initial CAD geometry was taken to be the upper $10 \%$ of the femur length parameter. This initial length is 11.33 inch and is identified as the distance between the point on the femoral head with the lowest $y$-value (see Fig.1 for orientation) and the bottom surface. The bottom surface was than sliced using a plane normal to the $y$-axis and a distance of 1.03 in the negative $y$ direction. Figure 2 below illustrates the sliced volume of the femur.

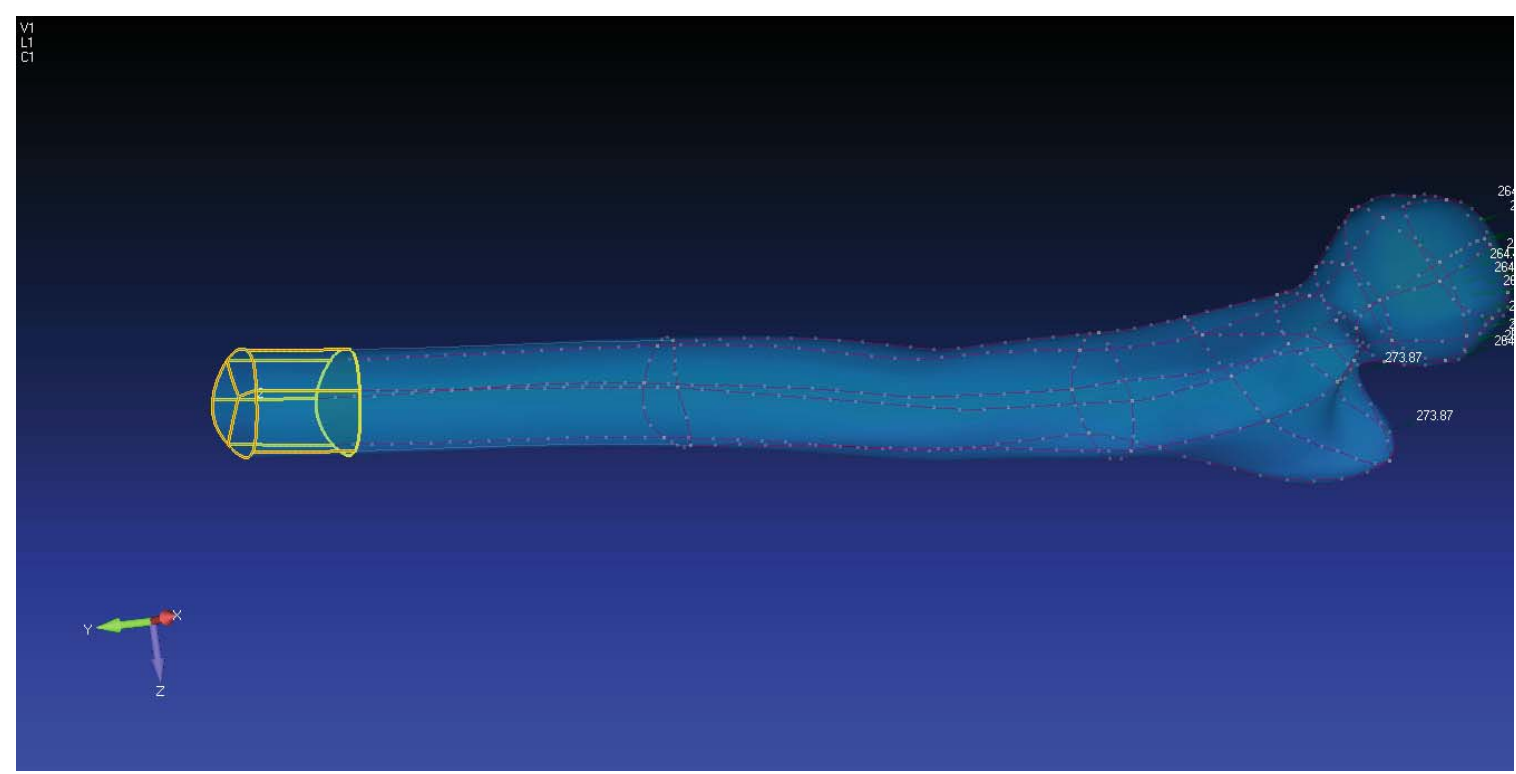

Fig.2. Sliced volume of the femur shown in a yellow outline. 
This leftover volume has a femoral length of 10.3 inch and will be the standard geometry of the CAD model. The lower $10 \%$ of the femoral length of 9.27 inch is achieved by repeating the process and shortening the femur's length.

\subsection{Mesh setup}

The pre and post processor for the finite element model is Femap Version 10.1. This software has advanced meshing algorithms which will be useful for the CAD geometry due to the complicated model. The femur, like most bone, is generally defined to have a hard cortical region that resides around the entire surface and a soft, porous trabecular region that fills in the center. The model will be meshed to account for both of these regions by first meshing the surfaces of the femur using triangular plate elements with the cortical material properties and then meshing the inside of the femur with solid tetrahedral elements based off the surface elements. The tetrahedral elements will represent the trabecular region of the femur.

The mesh of the femur will remain constant for all variables except for the length of the femur and the element size. The element size is adjusted by setting the desired value on the surfaces and volume and remeshing the CAD model. The thickness of the plate elements located on the surface of the femur will define the cortical thickness.

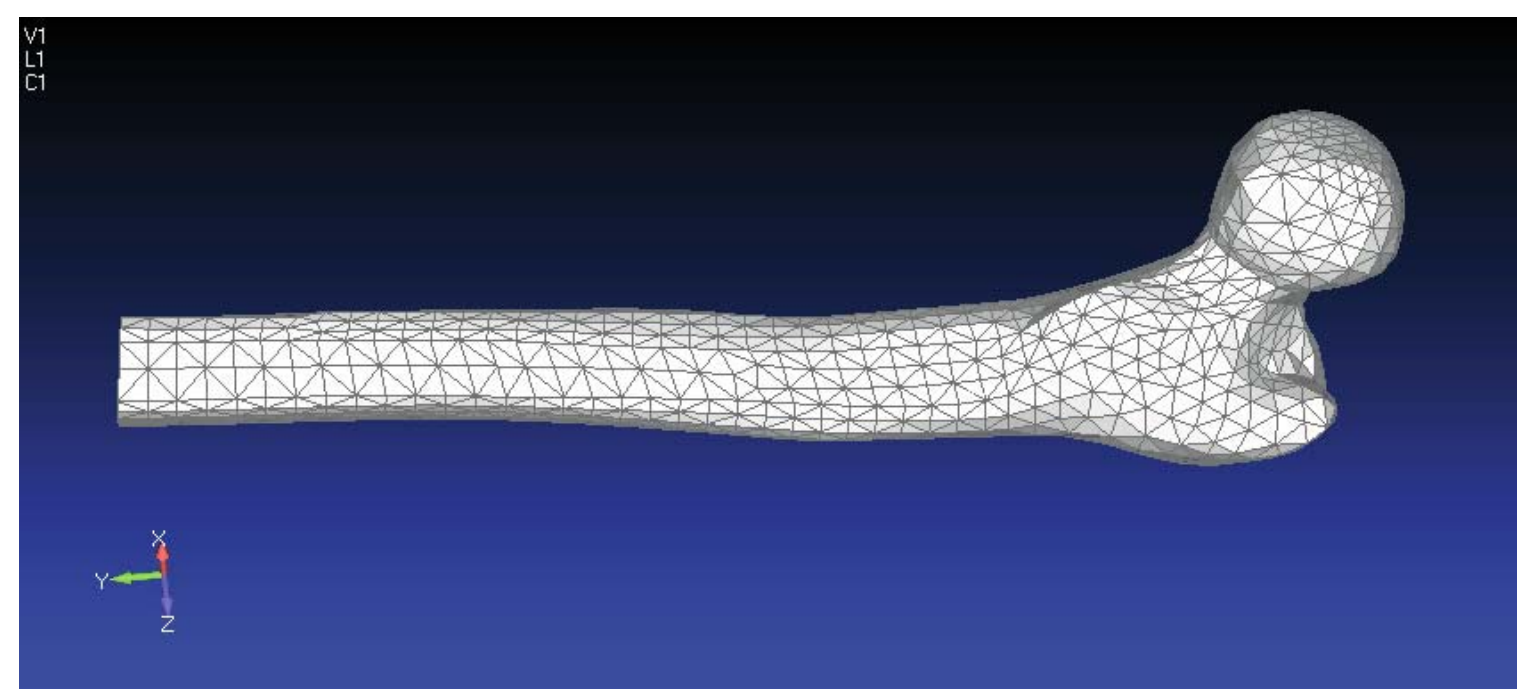

Fig.3. Standard mesh of the proximal femur.

\subsection{Boundary conditions}

\subsubsection{Loads}

The model is loaded with three different load cases based on the methods provided by Hazelwood's marathon training article for normal activity. Each set of loads is applied in two locations and are defined by the magnitude of the force and the angle it is applied with respect to the length of the femur. The three load cases are defined as Single Leg Stance, Abduction, and Adduction. The loads are applied directly on the geometry and adjusted so the uniform line load or surface load that is applied is equivalent to the single value force load. The location of the loading is shown in Fig.4 below. 


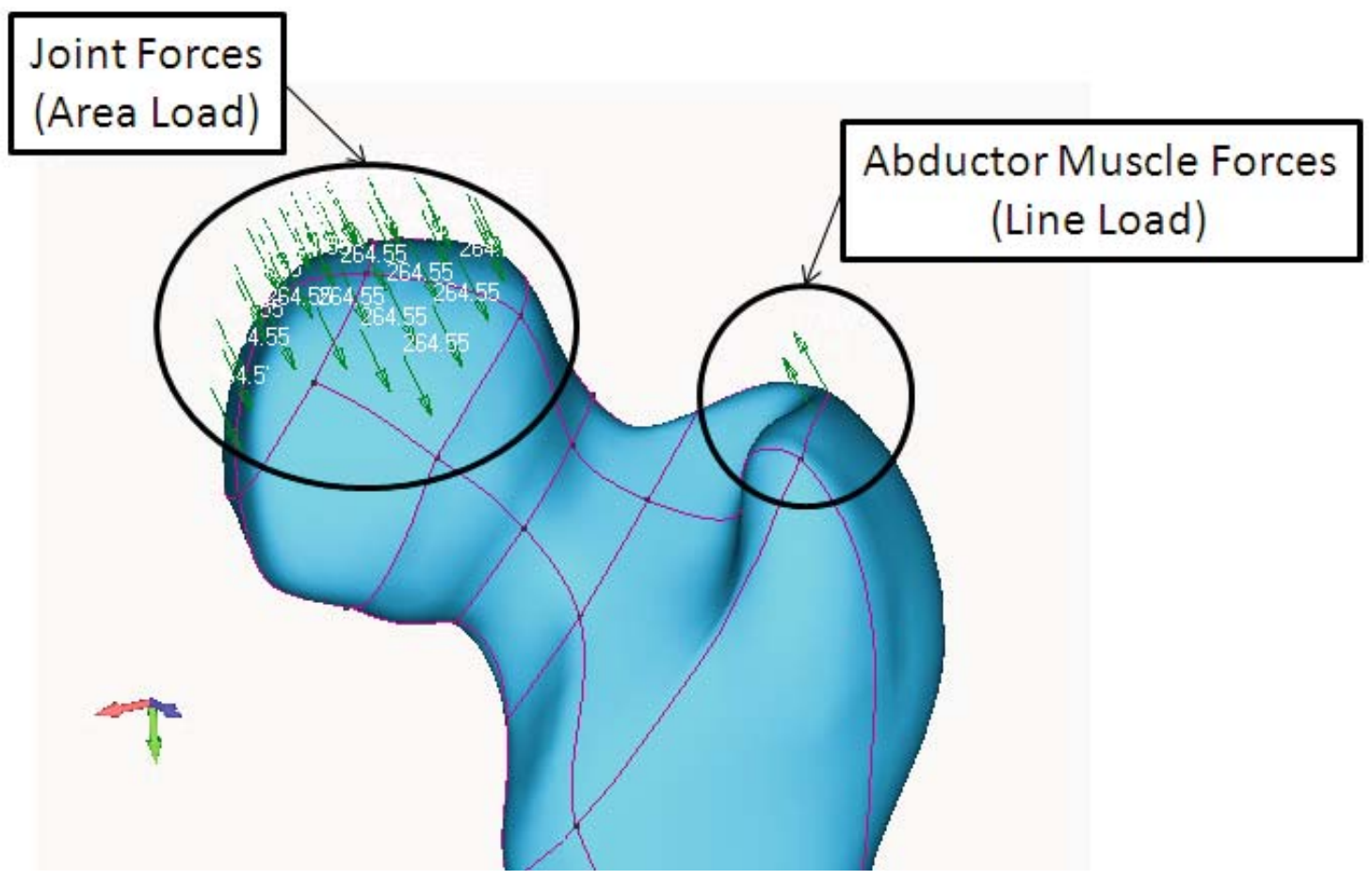

Fig.4. Location of applied loads.

Table 2 below summarizes the values and angles of the three loads. The joint force load is applied to an area of 1.97 inch which is obtained using the CAD geometry. The abductor muscle load is applied to a curve length of 1.38 inch which is also obtained through CAD geometry.

Table 2. Summary of the applied loads, geometric equivalent load, and angle.

\begin{tabular}{|r|r|r|r|}
\hline & Single Load (lb) & Geometric Eq. (lb) & Angle (deg) \\
\hline Joint forces & & & 264.5 \\
\hline Single Leg Stance & 520.9 & 132.2 & -15 \\
\hline Abduction & 260.3 & 176.7 & 56 \\
\hline Adduction & 348.0 & & \\
\hline Abductor Muscle Forces & & & \\
\hline Single Leg Stance & 378.8 & 273.9 & 28 \\
\hline Abduction & 189.3 & 136.9 & -8 \\
\hline Adduction & 253.1 & 183.0 & 65 \\
\hline
\end{tabular}

\subsubsection{Constraints}

The femur is constrained at the bottom of the femur where the length is truncated. The bottom surface of the femur is pinned to prevent translation in the $X, Y$, and $Z$ direction. The figure below shows the location of the surface that has the pinned constraint. 


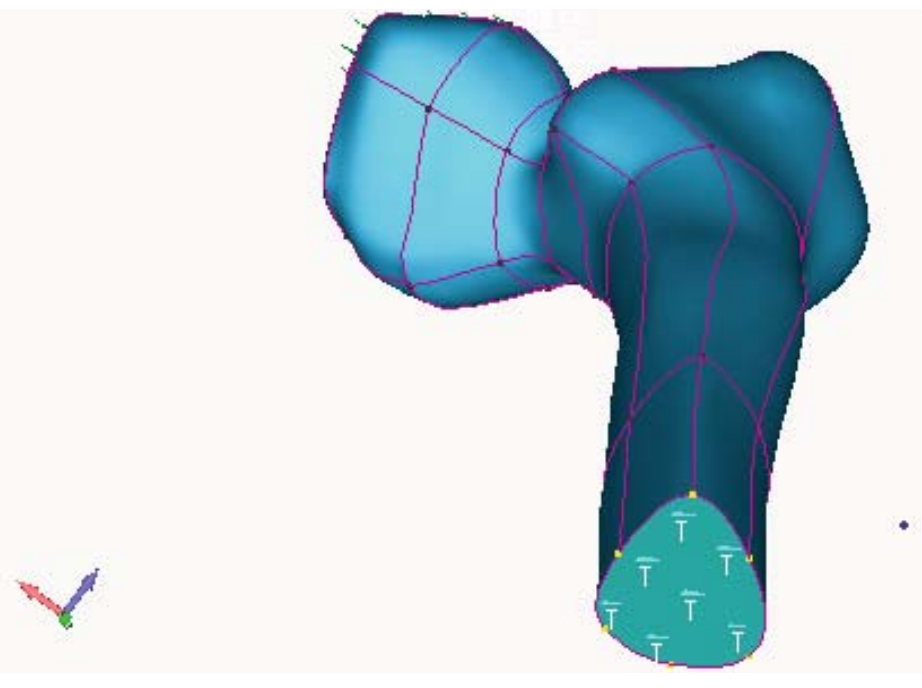

Fig.5. Location of the constrained surface.

\subsection{FEA results}

The model was run in NX NASTRAN v6.0 for all three load cases for a linear stress analysis which produces three outputs. Below are the contours of the maximum principal strain for the standard model femur and the three load cases.

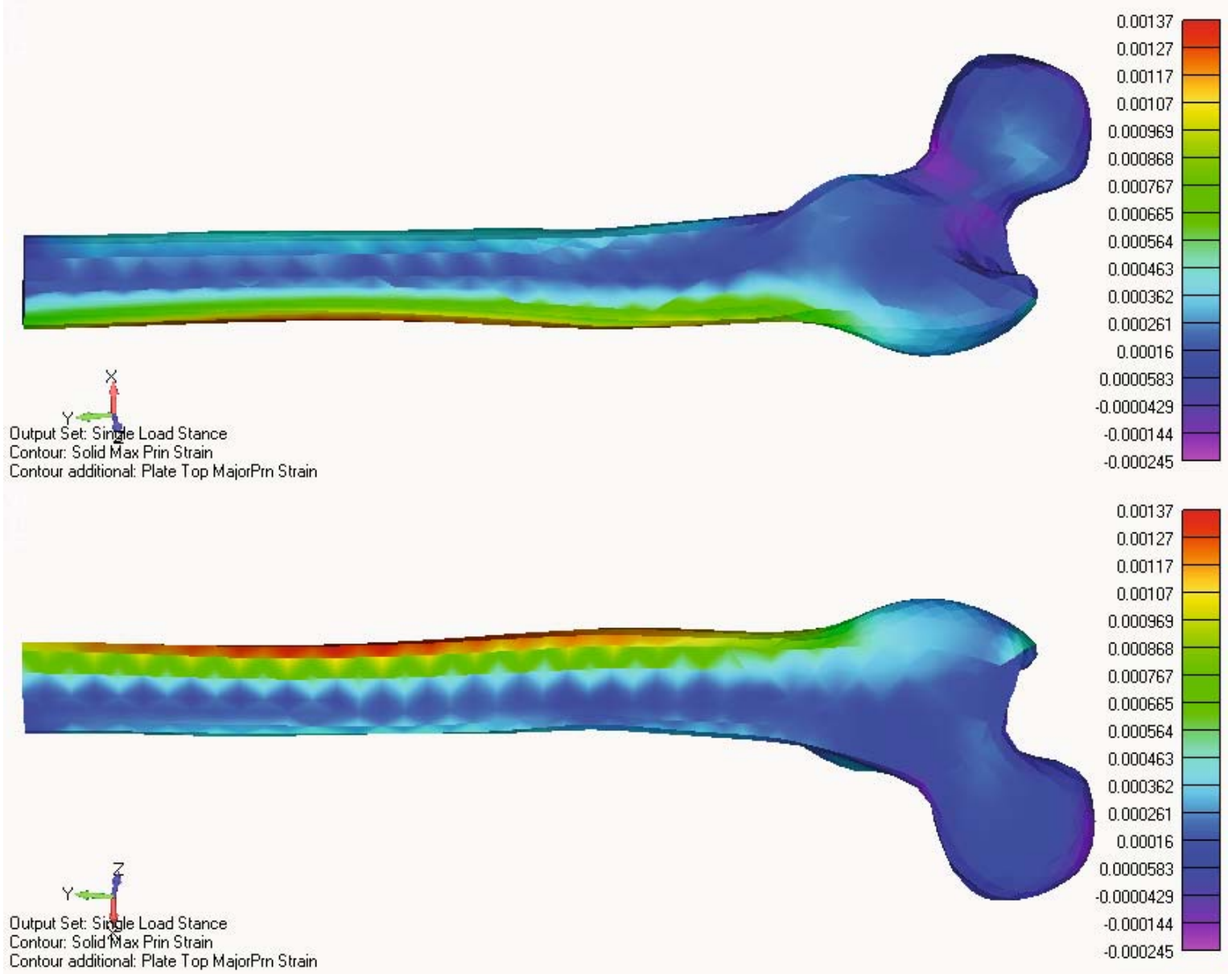

Fig.6. Front and back contours of the maximum principal strain for Single Load Stance load case. 


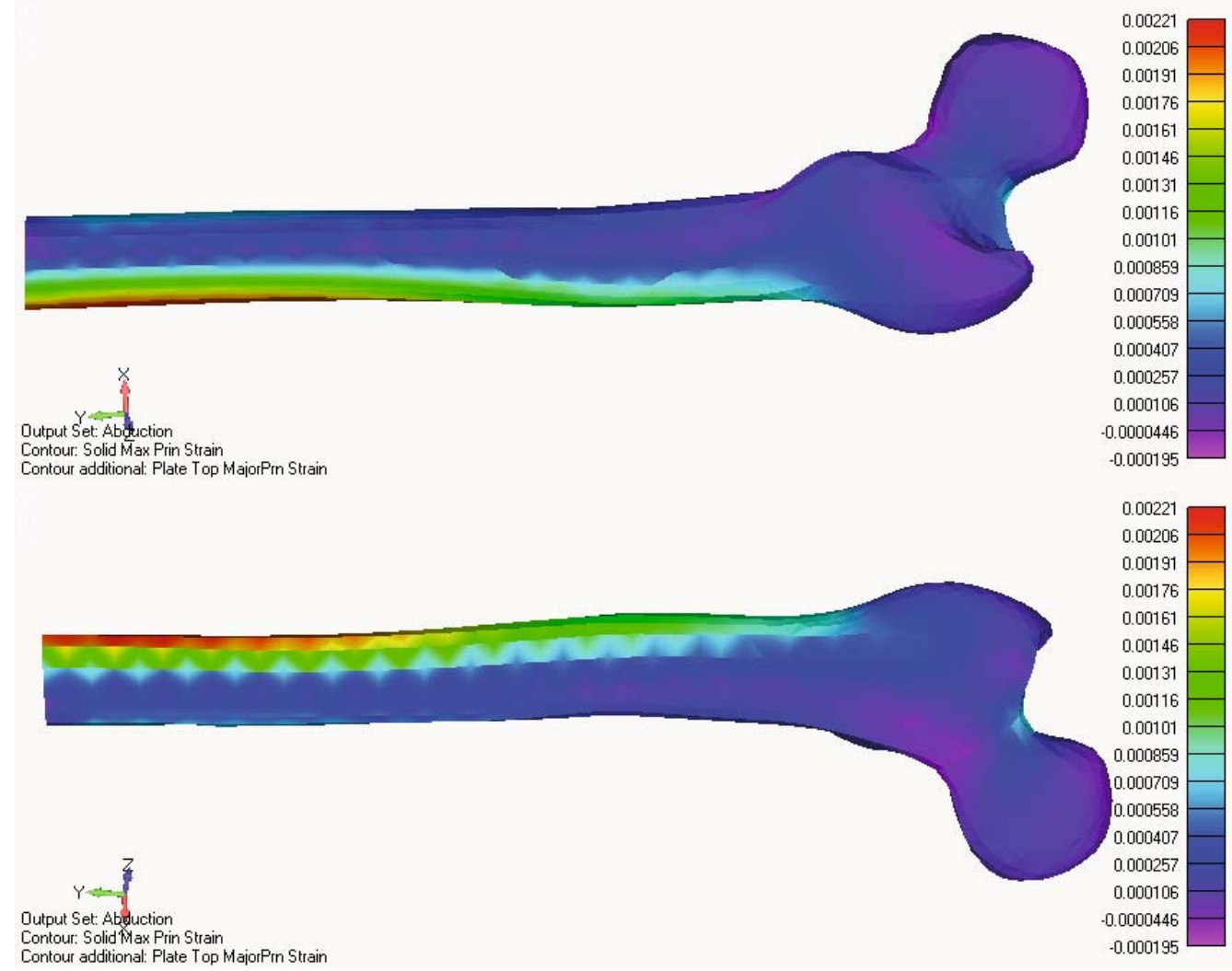

Fig.7. Front and back contours of the maximum principal strain for the Abduction load case.

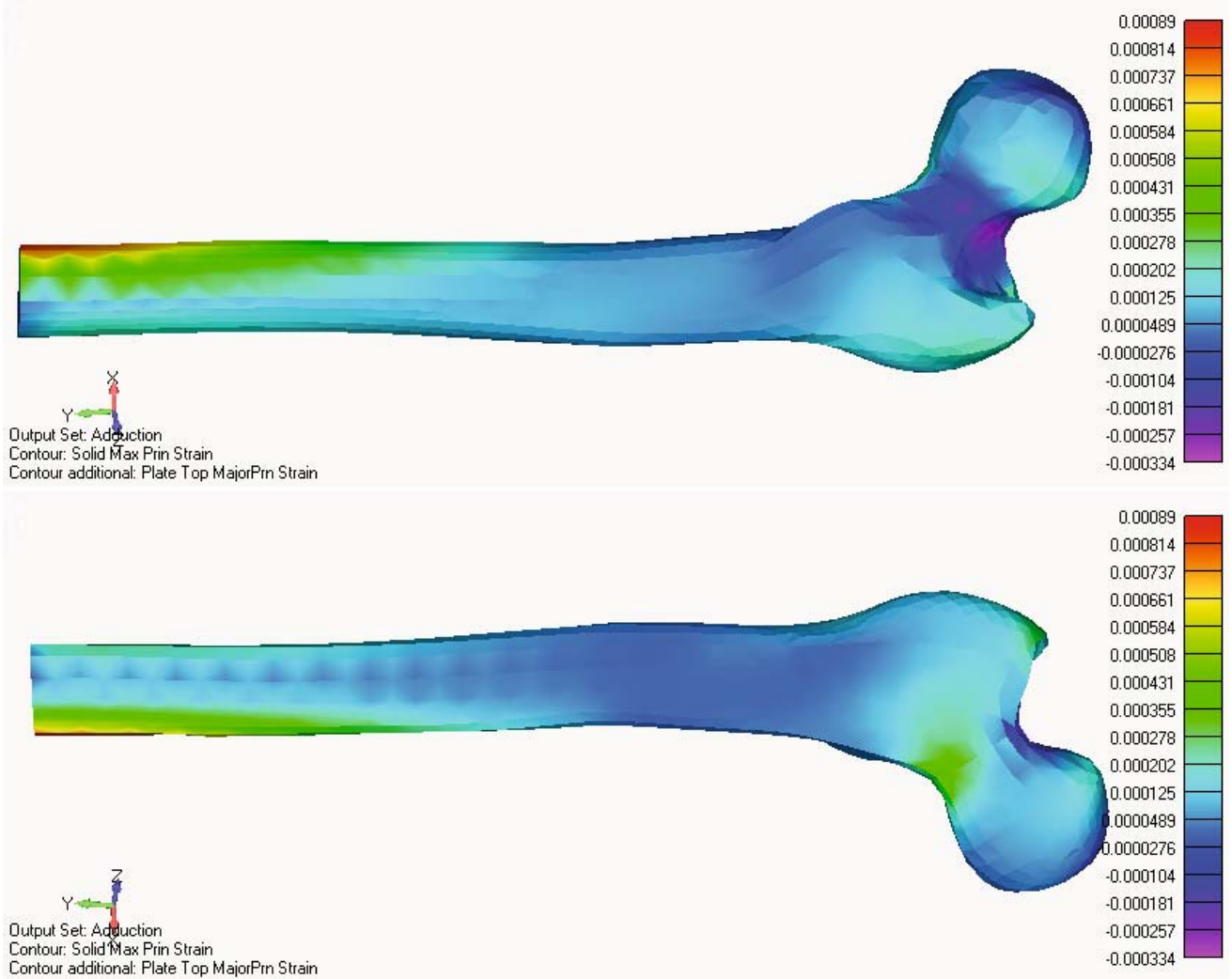

Fig.8. Front and back contours of the maximum principal strain for the Adduction load case. 


\subsection{Post processing}

After the results have been obtained, the maximum principal strains for each element and load case are exported from Femap to calculate the mechanical stimulus. The formula for the maximum principal strain will be used from Hazelwood's marathon training article. The mechanical stimulus is calculated for each element by using the formula below

$$
\Phi=\sum_{i=1}^{n} s_{i}^{q} * R_{L i}
$$

where:

$\Phi=$ mechanical stimulus,

$s=$ maximum principal strain,

$R_{L}=$ number of cycles per day,

$q=$ an exponent which is set to 4 .

The maximum principal strain is extracted from FEA and the number of cycles per day is estimated to be 4500 for the Single Leg Stance load case and 750 for both the Abduction and Adduction load cases.

For example, a randomly selected element from the standard model analysis (element \# 23662) will be used for a sample calculation of the mechanical stimulus.

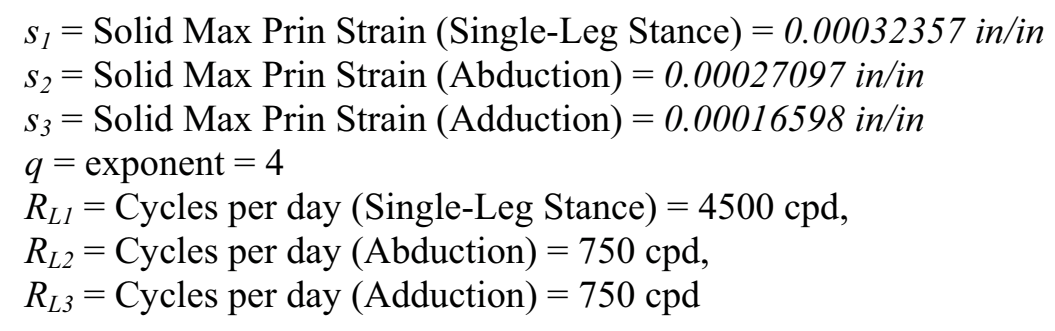

$$
\begin{aligned}
& \Phi=\left(s_{1}^{4} * R_{L 1}\right)+\left(s_{2}^{4} * R_{L 2}\right)+\left(s_{3}^{4} * R_{L 3}\right) \\
& \Phi=\left(0.00032357^{4} * 4500\right)+\left(0.00027097^{4} * 750\right)+\left(0.00016598^{4} * 750\right) \\
& \Phi=5.39397 \times 10^{-11} c p d, \\
& \Phi=5.39397 \times 10^{-11} \text { cycles per day. }
\end{aligned}
$$

Because the mechanical stimulus is a linear combination of the three different load cases, the element with the largest maximum principal strain from a specific load case may not have the largest mechanical stimulus. This means that each individual element requires its own calculation of mechanical stimulus and the largest mechanical stimulus is used as the maximum value to conduct the probabilistic analysis. Table 3 below summarizes the maximum mechanical stimulus for each varied parameter. 
Table 3. Summary of mechanical stimulus by parameter.

\begin{tabular}{|l|r|r|r|}
\hline Varying Properties & $-10 \%$ & Standard & $+10 \%$ \\
\hline Cortical Modulus (psi) & $4.3294723 \mathrm{E}-08$ & $2.8779578 \mathrm{E}-08$ & $1.9870871 \mathrm{E}-08$ \\
\hline Cortical poisson's ratio & $2.8740110 \mathrm{E}-08$ & $2.8779578 \mathrm{E}-08$ & $2.8839467 \mathrm{E}-08$ \\
\hline Trabecular Modulus (psi) & $2.9124360 \mathrm{E}-08$ & $2.8779578 \mathrm{E}-08$ & $2.8445521 \mathrm{E}-08$ \\
\hline Trabecular poisson's ratio & $\mathrm{NA}$ & $2.8779578 \mathrm{E}-08$ & $\mathrm{NA}$ \\
\hline Average Element Size (in) & $2.3924123 \mathrm{E}-08$ & $2.8779578 \mathrm{E}-08$ & $2.9886192 \mathrm{E}-08$ \\
\hline Cortical Thickness (in) & $4.0735081 \mathrm{E}-08$ & $2.8779578 \mathrm{E}-08$ & $2.1069250 \mathrm{E}-08$ \\
\hline Femur Length (in) & $2.9163445 \mathrm{E}-08$ & $2.8779578 \mathrm{E}-08$ & $2.9134099 \mathrm{E}-08$ \\
\hline Single Leg Stance cpd & $2.7354450 \mathrm{E}-08$ & $2.8779578 \mathrm{E}-08$ & $3.0305049 \mathrm{E}-08$ \\
\hline Single Leg Stance Load Joint (lb) & $4.5824658 \mathrm{E}-08$ & $2.8779578 \mathrm{E}-08$ & $2.0770432 \mathrm{E}-08$ \\
\hline Single Leg Stance Angle Joint (deg) & $6.1692841 \mathrm{E}-08$ & $2.8779578 \mathrm{E}-08$ & $1.9454512 \mathrm{E}-08$ \\
\hline Single Leg Stance Load Abductor (lb) & $1.9752424 \mathrm{E}-08$ & $2.8779578 \mathrm{E}-08$ & $5.6820696 \mathrm{E}-08$ \\
\hline Single Leg Stance Angle Abductor (deg) & $2.0451879 \mathrm{E}-08$ & $2.8779578 \mathrm{E}-08$ & $4.8349026 \mathrm{E}-08$ \\
\hline Abduction cpd & $2.7427131 \mathrm{E}-08$ & $2.8779578 \mathrm{E}-08$ & $3.0232106 \mathrm{E}-08$ \\
\hline Abduction load Joint (Ib) & $2.3764790 \mathrm{E}-08$ & $2.8779578 \mathrm{E}-08$ & $3.8188272 \mathrm{E}-08$ \\
\hline Abduction Angle Joint (deg) & $2.5695616 \mathrm{E}-08$ & $2.8779578 \mathrm{E}-08$ & $3.3707560 \mathrm{E}-08$ \\
\hline Abduction Load Abductor (lb) & $2.9566544 \mathrm{E}-08$ & $2.8779578 \mathrm{E}-08$ & $2.8119942 \mathrm{E}-08$ \\
\hline Abduction Angle Abductor (deg) & $3.0383254 \mathrm{E}-08$ & $2.8779578 \mathrm{E}-08$ & $2.7448261 \mathrm{E}-08$ \\
\hline Adduction cpd & $2.8779539 \mathrm{E}-08$ & $2.8779578 \mathrm{E}-08$ & $2.8779618 \mathrm{E}-08$ \\
\hline Adduction Load Joint (Ib) & $2.8779185 \mathrm{E}-08$ & $2.8779578 \mathrm{E}-08$ & $2.8786766 \mathrm{E}-08$ \\
\hline Adduction Angle Joint (deg) & $2.8779185 \mathrm{E}-08$ & $2.8779578 \mathrm{E}-08$ & $2.8784695 \mathrm{E}-08$ \\
\hline Adduction Load Abductor (Ib) & $2.8784762 \mathrm{E}-08$ & $2.8779578 \mathrm{E}-08$ & $2.8779185 \mathrm{E}-08$ \\
\hline Adduction Angle Abductor (deg) & $2.8780754 \mathrm{E}-08$ & $2.8779578 \mathrm{E}-08$ & $2.8779279 \mathrm{E}-08$ \\
\hline
\end{tabular}

It should be noted that the Poisson's ratio of the trabecular material property did not show any change in the mechanical stimulus when the parameter was varied + and $-10 \%$. Because there was no change in the mechanical stimulus, it was determined that this parameter would not yield any results when processed through NESTEM and, thus, was left out of the analysis.

\subsection{Probabilistic analysis}

The parameters defined in Tabs 1 and 3 were submitted to the NESTEM probabilistic analysis program to determine the sensitivity of each parameter. The non-linear analysis program generated productive results that helped determine the sensitivities of the parameters at various probabilities. Figure 9 below shows the probability as a function of the maximum mechanical stimulus that will occur within a femur using normal activity load scheduling. Figure 10 show the sensitivity of each parameter for a probability of 0.99 . 


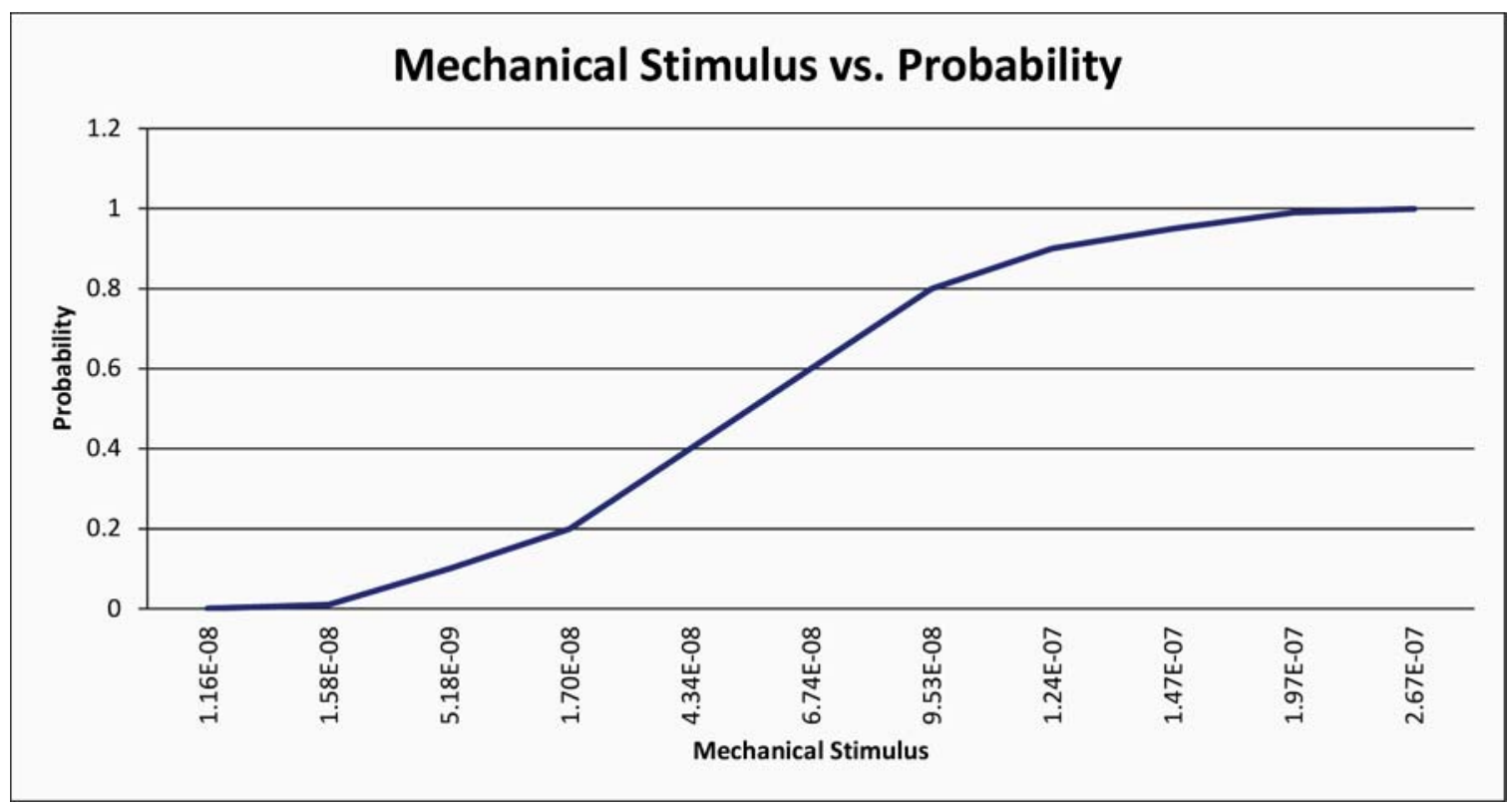

Fig.9. Plot of the mechanical stimulus vs. probability.

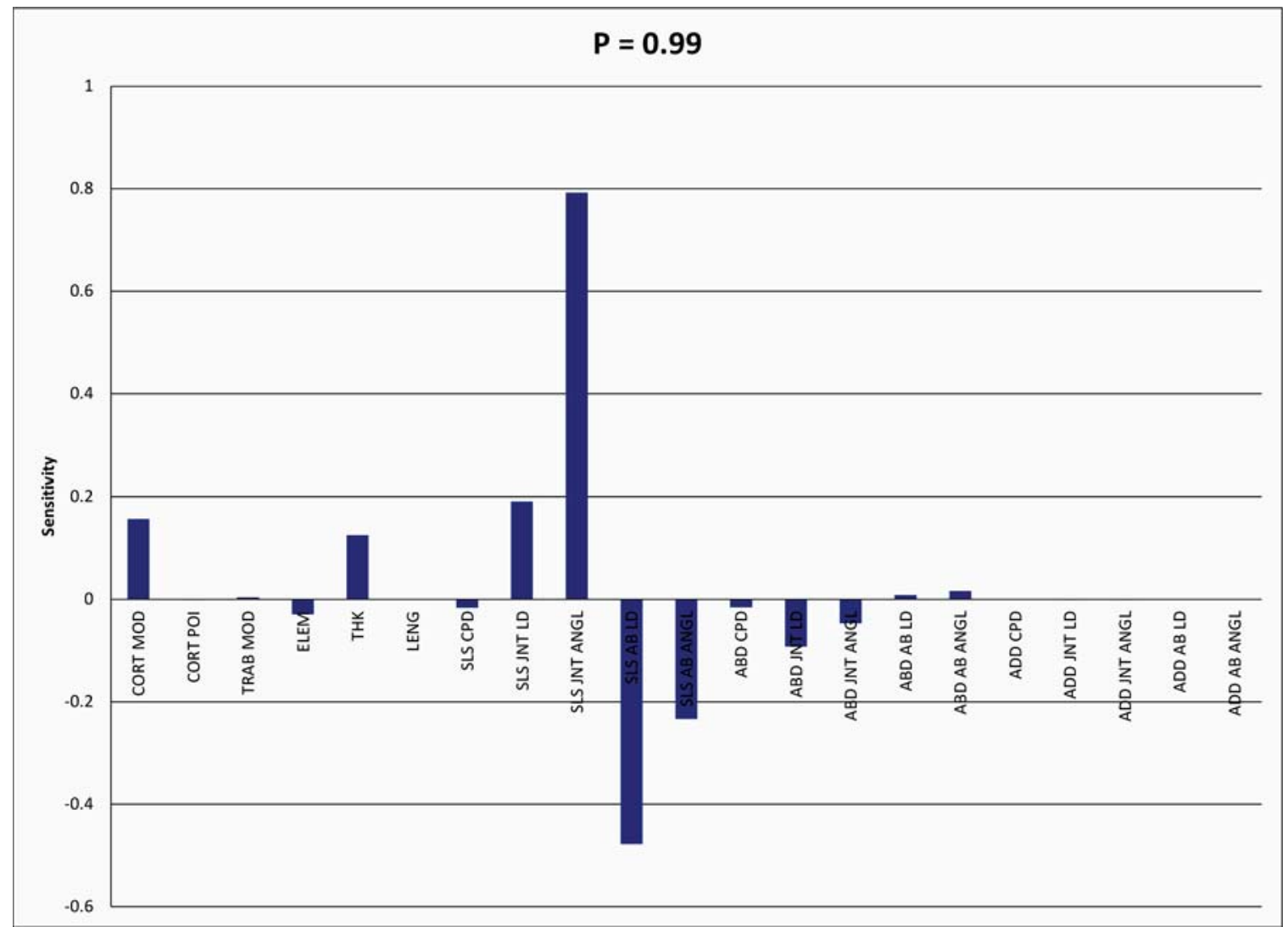

Fig.10. Sensitivities of each parameter with a probability of 0.99 . 


\section{Conclusion}

The material and geometric properties show that the stiffer components have the greatest sensitivities. The two specific parameters that have the highest sensitivity with a probability of 0.999 are the modulus of elasticity of the cortical bone and the thickness of the cortical region. The probabilistic analysis shows that the larger strains are occurring in the cortical regions which are modeled as plate elements on the outer surfaces of the femur.

The probabilistic analysis also shows general trends that the sensitivities of each group of parameters for a given load case behave similarly. For example, the group of parameters associated with the Single Leg Stance has a much higher sensitivity than the parameters associated with the Adduction load case. This can be explained by the calculation of the mechanical stimulus performed during post processing and that the Adduction load case does not produce strains in the bone that are as large as the ones produced for the other two load cases. The cycles per day parameter also contributes to the vast differences in sensitivity between the load cases because the value for Single Leg Stance is assumed to be 4500 cycles per day while Abduction and Adduction are only 750 cycles per day.

The sensitivity analysis using NESTEM also shows that the angle of Single Leg Stance has the most influence on the maximum value of the mechanical stimulus with a probability of 0.999 . This angle parameter can directly affect the moment that the joint load creates because the femoral head is offset from the length of the femur. The joint angle may also correlate to the way a person stands and the position of their feet in the standing position. This analysis provides a great example of how the finite element model coupled with the probabilistic analysis can help identify how the parameters in the loading can affect the mechanical stimulus which is used to characterize the bone remodeling process.

\section{References}

Hazelwood S.J., Martin R.B., Rashid M.M. and Rodrigo J.J. (2001): A mechanistic model for internal bone remodeling exhibits different dynamic responses in disuse and overload. - Journal of Biomechanics, vol.34(3), pp.299-308

Hazelwood S.J. and Castillo A.B. (2007): Simulated effects of marathon training on bone density, remodeling, and microdamage accumulation of the femur. - International Journal of Fatigue, vol.29, pp.1057-1064.

Received: June 18, 2012

Revised: May 5, 2013 